\title{
Lung cancer and mesothelioma in the pleura and peritoneum among Swedish insulation workers
}

\author{
Bengt Järvholm, Åke Sandén
}

\begin{abstract}
Objectives-To estimate the risk of cancer and death in Swedish insulation workers some years after their exposure to asbestos had stopped. One hypothesis was that the risk of lung cancer would tend to decrease some years after the exposure had ended.

Methods-In a cohort study the cancer morbidity and cause of death was investigated in 248 insulation workers and compared with the corresponding morbidity and mortality in the general population. Due to stringent regulations, exposure to asbestos of all types had almost ended in Sweden in the mid-1970s. Through a questionnaire, surviving insulation workers were asked about their exposure to asbestos and their smoking habits.

Results-Between 1970 and 1994 there were 86 deaths compared with the 46.0 expected (standardised incidence ratio (SIR) $1.9 ; 95 \%$ confidence interval $(95 \%$ CI) 1.5 to 2.3 ), the increase was mainly due to an increased cancer mortality. The morbidity was increased for lung cancer (11 cases $v 2.5$ expected (SIR 4.4; 95\% CI 2.2 to 7.9)), peritoneal mesothelioma (seven cases; no expected incidence could be calculated as the occurrence is too rare in the general population), cancer in pancreas (five cases $v 0.7$ expected (SIR 7.1; $95 \%$ CI 2.3 to 16.7$)$ ). No cases of pleural mesothelioma were found. The risk of lung cancer did not tend to approach that of the general population after the exposure to asbestos decreased.

Conclusions-In the 1980 s and the early 1990s, Swedish insulation workers still have a highly increased risk of diseases related to asbestos. The attributable risk for death and cancer was about $50 \%$. The study also confirms the previous finding that mesothelioma in insulation workers seems to be situated in the peritoneum more often than in the pleura.

(Occup Environ Med 1998;55:766-770)
\end{abstract}

Keywords: asbestos; lung cancer; mesothelioma

The risk for cancer after exposure to asbestos varies for different occupational groups. ${ }^{1}$ Both the degree of risk and the distribution between different types and sites of tumours varies. Most studies have found a high ratio of pleural to peritoneal mesotheliomas, but a few studies have shown a higher incidence of peritoneal than pleural mesotheliomas-for example, among insulation workers. ${ }^{2}$ In a study of ship- yard workers in Göteborg, we found an increased risk of pleural mesothelioma but no cases of peritoneal mesothelioma. ${ }^{3}$ Some years after the exposure to asbestos had ended, the risk of lung cancer among those shipyard workers was not increased, yet they had an increased risk of pleural mesothelioma, indicating that asbestos mainly acts as a promotor in the carcinogenesis of lung cancer.

The objective of this study was to investigate the occurrence of lung cancer and mesothelioma in Swedish insulation workers, at a time some years after the exposure to asbestos had stopped. Furthermore, we use the results to discuss possible mechanisms for the carcinogenic effect of asbestos. This study is a follow up to a previously published study which detailed the risk of cancer during the 1970s. ${ }^{4}$

\section{Subjects and methods}

This is a historical cohort study in which cancer morbidity and mortality in insulation workers was compared with that of the general population.

A former union representative listed all insulation workers affiliated with the Construction Workers Association in Göteborg in the late 1960s. This list contained 272 people, all men. Three men were on the list twice. The men on this list constituted the exposed workers in this cohort study. The list contains no information other than the names and personal numbersfor example, no information about employment time, smoking habits, or exposure to asbestos.

By linking the insulation workers' personal numbers with the death register and cancer register of Sweden, the number of deaths, cause of death, and morbidity from cancer were found. The cancer register was established in 1958 , and the death register has been computerised since 1952. At the time of the linkage, the causes of death could be obtained up to 1994, and the cancer morbidity up to 1992 .

To ensure the completeness of the follow up, the personal numbers were also linked to the register of the population presently living in Sweden, and the register of emigrants, where one man was found. Sixteen people were not found in any of the registers and were excluded from the analysis.

The study was approved by the Committee of Ethics at the university and by the Data Inspectorate. Approval from the Data Inspectorate required that all living insulators be asked whether they agreed to participate in the linkage. A letter was sent to all of the living men and five insulation workers notified us and said that they wanted to be removed from the regis- 
Table 1 Year of birth of the insulators

\begin{tabular}{lc}
\hline Year of birth & $n$ \\
\hline $1901-9$ & 18 \\
$1910-9$ & 41 \\
$1920-9$ & 46 \\
$1930-9$ & 59 \\
$1940-9$ & 80 \\
$1950-2$ & 4 \\
Total & 248 \\
\hline
\end{tabular}

ter. There was no obligation to ask relatives of deceased workers and consequently all deceased workers were included in the analysis.

During the study, the Data Inspectorate informed us that the computerised register of the insulation workers should not be destroyed but may be stored at the national archives. However, we were also requested to inform the living workers about this decision. We therefore sent the living men a letter about the storage. We also included a short questionnaire about smoking habits, the use of asbestos, and the types of asbestos they had used. The questionnaire was sent to 173 workers and 98 (57\%) replied. No one refused to be included in the register that would be stored. In total, the cohort consists of 248 men (269 minus five refusers and 16 not found in registers).

The expected cancer incidence and mortality were calculated by the person-year method. ${ }^{5}$ Each man contributed person-years from 1970 until the year of death, the year of emigration, or until the age of 84 . Mortalities and cancer incidences stratified for calendar year (one year interval), age group (5 year intervals) and sex for the general population were available from the death register and cancer register respectively. The national death register contains the underlying cause of death and contributing causes of death. The national cancer register started in 1958 and reporting is mandatory for all causes of cancer at the time of diagnosis.

Ninety five per cent confidence intervals (95\% CIs) of risk ratios (RRs) were calculated assuming a poisson distribution. The study is small; the expected number of lung cancers in the more recent period is $\simeq 2$ and only RRs of $\geqslant 4$ can be determined with a power of 0.8 . However, the expected number of incident cases of cancer of all sites is about 15 for the more recent period and an $R R$ of $\geqslant 2$ can be detected with a power of 0.8 .

\section{Results}

The years of birth of the insulation workers are presented in table 1, the oldest workers were born in 1901 and the youngest in 1952. The usual time of retirement in the late 1960s was 67 years.

The mortality among the insulation workers was higher than in the general population between 1970 and 1994 (RR 1.9; 95\% CI 1.5 to 2.3), mainly due to a highly increased risk of cancer (table 2). However, the mortality in non-malignant lung disease was significantly increased, seven cases versus 2.4 expected. There was just one case of asbestosis as a underlying cause of death. The other causes of death in non-malignant lung diseases were five cases of pneumonia and one case of asthma. The mortality from all causes increased less in 1970-9 than in 1980-94, but the precision of the estimates is not sufficient to exclude a similar risk, table 1.

An analysis of cancer morbidity showed significantly increased incidences of bronchial cancer (RR 4.4, 95\% CI 2.2 to 7.9), peritoneal mesothelioma, and pancreatic cancer (table 3 ). However, there was no case of pleural mesothelioma. In the cancer register five of the bronchial cancers were classified as squamous cell carcinomas, one as adenocarcinoma, four as oat cell cancers, and one was not classified. The standardised rate ratios (SRRs) were calculated for cancer of all sites and bronchial cancer based on the age distribution of personyears for the total period 1970-92 to make a comparison between the two periods 1970-9 and 1980-92. The SRRs were 4.0 (95\% CI 0.8 to 11.7$)$ and 5.0 (95\% CI 2.1 to 9.8$)$ respectively for lung cancer and 1.5 (95\% CI 0.7 to 2.7$)$ and 1.4 (95\% CI 0.9 to 2.0$)$ for all sites. The number of peritoneal mesotheliomas in the general population are too few for a SRR analysis. The crude incidences were similar for the two periods (1.3 and 1.6 cases per 1000 person-years respectively (table 3 )). Between 1980 and 1992 the attributable risk for all causes of death was $53 \%$, and $52 \%$ for all sites of cancer.

Based on the questionnaire, $19 \%$ of the men answered that they were smokers, $55 \%$ exsmokers, and $25 \%$ non-smokers.

\section{EXPOSURE}

In Sweden the use of asbestos, including chrysotile, was strictly regulated in the mid-1970s. The regulation resulted in a drop in the importation of raw asbestos to Sweden to drop from about 18 000-200 00 tonnes in the 1960s and beginning of the 1970 s to around 1000 tonnes, in the late 1970s.

Table 2 Mortality in the insulators in 1970-9 and 1980-94 respectively

\begin{tabular}{|c|c|c|c|c|c|c|}
\hline \multirow[b]{2}{*}{ Cause of death $(I C D-8)$} & \multicolumn{3}{|l|}{$1970-9$} & \multicolumn{3}{|l|}{ 1980-92 } \\
\hline & Observed & Expected & $\operatorname{SIR}(95 \% C I)$ & Observed & Expected & $\operatorname{SIR}(95 \% C I)$ \\
\hline Tumours (140-209) & 11 & 3.2 & $3.4(1.7-6.2)$ & 30 & 8.3 & $3.6(2.4$ to 5.2$)$ \\
\hline Cardiovascular diseases (390-458) & 4 & 6.2 & $0.7(0.2-1.7)$ & 22 & 16.8 & $1.3(0.8$ to 2.0$)$ \\
\hline Non-malignant lung diseases & & & & & & \\
\hline$(460-519)$ & 1 & 0.5 & $2.0(0.1-11.1)$ & 6 & 1.9 & $3.2(1.2$ to 6.9$)$ \\
\hline Accidents (800-999) & 1 & 2.1 & $0.5(0.0-2.7)$ & 1 & 2.6 & $0.4(0.0$ to 2.1$)$ \\
\hline Other & 4 & 3.2 & $1.3(0.3-3.2)$ & 6 & 1.2 & $5.0(1.8$ to 10.9$)$ \\
\hline All & 21 & 15.2 & $1.4(0.9-2.1)$ & 65 & 30.8 & $2.1(1.6$ to 2.7$)$ \\
\hline Number of person-years & 2330.5 & & & 2544.0 & & \\
\hline
\end{tabular}


Table 3 Cancer morbidity among the insulators in 1970-9 and 1980-92

\begin{tabular}{|c|c|c|c|c|c|c|}
\hline \multirow[b]{2}{*}{ Site $(I C D-8)$} & \multicolumn{3}{|l|}{$1970-79$} & \multicolumn{3}{|l|}{ 1980-92 } \\
\hline & Observed & Expected & $\operatorname{SIR}(95 \% C I)$ & Observed & Expected & $\operatorname{SIR}(95 \% C I)$ \\
\hline Nose and pharynx (140-149) & 0 & 0.2 & $0(0$ to 18$)$ & 0 & 0.4 & $0(0$ to 9.2$)$ \\
\hline Gastrointestinal tract (150-159) & 5 & 1.7 & $2.9(0.95$ to 6.9$)$ & 8 & 3.6 & $2.2(0.96$ to 1.4$)$ \\
\hline Pancreas (157) & 2 & 0.2 & $10.0(1.2$ to 36$)$ & 3 & 0.5 & $6.0(1.2$ to 18$)$ \\
\hline Peritoneal mesothelioma (159) & 3 & —夫 & & 4 & 一 $^{\star}$ & \\
\hline Lung (162) & $3 \dagger$ & 0.8 & $3.8(0.8$ to 11$)$ & $8 \ddagger$ & 1.7 & $4.7(2.0$ to 9.3$)$ \\
\hline Pleural mesothelioma (163.0) & 0 & —* & & 0 & —^ & \\
\hline Prostate (185) & 0 & 0.9 & $0(0$ to 4.1$)$ & 3 & 3.0 & $1.0(0.2$ to 2.9$)$ \\
\hline Kidney and urinary bladder (188 to 189 ) & 0 & 0.8 & $0(0$ to 4.6$)$ & 4 & 1.7 & $2.4(0.6$ to 60$)$ \\
\hline Brain (191 to 192$)$ & 0 & 0.3 & $0(0$ to 12$)$ & 2 & 0.5 & $4.0(0.5$ to 14$)$ \\
\hline Lymphoma (200 to 202 ) & 1 & 0.4 & $2.5(0.1$ to 14$)$ & 0 & 0.8 & $0(0$ to 4.6$)$ \\
\hline Unknown (197 to 199$)$ & 0 & 0.2 & $0(0$ to 18$)$ & 3 & 0.5 & $6.0(1.2$ to 18$)$ \\
\hline All & $10 \S$ & 6.4 & $1.6(0.8$ to 2.9$)$ & 319 & 14.8 & $2.1(1.4$ to 3.0$)$ \\
\hline Number of person-years & 2330.5 & & & 2544. 0 & & \\
\hline
\end{tabular}

*Incidence in Sweden is too low to make a calculation of expected rate feasible.

†Occurring in 1971, 1976, and 1979.

†Occurring in 1980, 1983, 1984, 1985, 1988 (3 cases) and 1990.

SExcept the mentioned sites there was one case of malignant melanoma.

IExcept the mentioned sites there was one case of malignant melanoma and one case of testicular cancer.

Twelve per cent of the insulation workers answering the questionnaire said that they had never worked with asbestos, $87 \%$ answered that they had worked with white asbestos (chrysotile), $51 \%$ with brown asbestos (amosite), and $43 \%$ with blue asbestos (crocidolite) sometimes or often. However, about $30 \%$ reported that they did not know if they had worked with brown or blue asbestos. Their mean (range) exposure time to asbestos was $14.4(0-46)$ years.

\section{Discussion}

This study shows that Swedish insulators have an increased risk of lung cancer several years after their exposure to asbestos has stopped. Furthermore, it shows that there is a considerably increased risk of peritoneal mesothelioma but there was no case of pleural mesothelioma.

ASPECTS OF VALIDITY

The loss of follow up in this cohort study is rather small and will probably not influence the results. The most probable reason for the loss to follow up is either wrong personal number or that the workers have emigrated without notifying the authorities. Insulation workers have often been employed by subcontractors. Some of the men may just have been temporary workers in Sweden. However, a loss to follow up due to death may have resulted in an underestimated risk. Thus, the findings may be conservative.

This is a small study with consequently low precision in point estimates of the RR. A moderately increased $R R,<2$, for lung cancer would be impossible to detect. One major hypothesis was that the risk of lung cancer would decrease some years after the exposure had decreased substantially, but even if the estimates have wide $95 \%$ CIs, there is no indication that the risk in the more recent period is similar to that of the general population (table 3).

According to the Data Inspectorate we had to notify all living insulation workers and inform them that we were establishing a computerised register including their personal numbers. Only those workers that notified us and told us that they did not want to be a member of the register were excluded. Those who did not reply were allowed to be included in the register. However, as those who were excluded were living the expected numbers will be somewhat underestimated for all causes of death. Also the expected morbidity of cancers is underestimated, especially for sites with a poor prognosis. However, only five people refused to be included and such a small exclusion will only marginally influence the expected rates.

All diagnoses were based on information received from the cancer register and death register. Studies of the cancer register have shown that the diagnoses are accurate. Mesotheliomas are only accepted in the register if they are based on histological examinations. Thus, the number of mesotheliomas may be underestimated through this procedure. The completeness of the register does seem to be high. A study by Mattson and Wallgren ${ }^{6}$ found that about $3 \%$ of the cancers of the trachea, bronchus, lung, and pleura were not reported. The studies of insulation workers from the United States have found that the number of mesotheliomas increased if all cases were reviewed by a trained pathologist. ${ }^{7}$ However, there should be no bias from exposure status as the same procedure is done with both the exposed insulation workers and the reference group, the general population.

\section{LUNG CANCER}

Our study shows an increased risk of lung cancer of similar size to another study of insulation workers; Selikoff et al found an RR of 4.6 in United States and Canadian insulation workers. There is no indication that the risks of lung cancer have decreased in the 1980s when the exposure of asbestos ended in Sweden. A study of Swedish shipyard workers from the same city as the insulation workers found no increased risk of lung cancer 7-15 years after the exposure to asbestos had stopped. ${ }^{3}$ Some other studies have also indicated that the risk of lung cancer may decrease after the exposure to asbestos has stopped. ${ }^{8}$

The mechanism for such a decrease may be that asbestos acts primarily as a promoter and cocarcinogen in causing lung cancer, and it also acts as an initiator in causing mesothelioma. ${ }^{9}$ If 
asbestos acts primarily as a cocarcinogen or promoter, the risk should decrease some years after the exposure has stopped. Thus, the risk of lung cancer after asbestos exposure has stopped seems to be different in the insulation workers and the shipyard workers. This difference may be due to the type of asbestos to which these workers have been exposed. Shipyard workers were mostly exposed to chrysotile whereas the insulation workers often worked with amphiboles. About $50 \%$ of the insulation workers that answered the questionnaire confirmed that they had been working either with amosite or crocidolite sometimes or often. However, shipyard workers had often had indirect exposure to amosite, which was used in spray insulation of the ships during the 1950s, 1960 s, and early 1970s. The spraying was done by subcontracted insulation workers.

The questionnaire in 1996 indicated that $19 \%$ of the insulation workers were current smokers. The percentage of daily smokers among Swedish men was between 14\% and $30 \%$ in 1995 in men born between 1911 and $1950 .{ }^{10}$ Thus, the current smoking habits of the insulation workers interviewed seem to be similar to the habits in the reference population. However, we only got information about smoking habits from 98 people and their smoking habits may be different from the dead workers and those who did not reply. The smoking habits could therefore be underestimated, but we think it is unlikely that heavy smoking habits would explain the highly increased risk in lung cancer.

PERITONEAL AND PLEURAL MESOTHELIOMA

Most studies of workers exposed to asbestos have indicated a higher risk of pleural than peritoneal mesothelioma. ${ }^{1}$ There were 108 male cases of pleural mesotheliomas in Sweden, and five cases of peritoneal mesothelioma in 1992. Other studies of insulation workers have indicated a higher risk of mesothelioma in the peritoneum than in the pleura. ${ }^{711} \mathrm{~A}$ study of American and Canadian insulation workers found 112 cases of peritoneal mesothelioma and 63 cases of pleural mesothelioma after reviewing and reclassifying the diagnosis. If the corresponding ratio between peritoneal and pleural mesothelioma had occurred in this study there would have been 3.9 cases of pleural mesothelioma but there were none. This difference is unlikely to be due to chance $(p=0.02)$. In a study in the London area, Browne and $\mathrm{Smith}^{2}$ found a similar number of peritoneal and pleural mesotheliomas in laggers. Applying a ratio of $1: 1$ seven cases of pleural mesothelioma would have been expected in our study $(p=0.001)$. In a study of Swedish shipyard workers from the same city there were 11 cases of pleural mesothelioma in 35155.8 person-years. If the same rate had been applied to this cohort of insulation workers there would have been 1.5 cases of pleural mesothelioma (4874.5 person-years (11 cases)/ 35155.8 person-years). The difference between no case and 1.5 expected is not unlikely $(p=0.2)$. The insulation workers in this cohort are somewhat older than the shipyard workers and the expected number should be higher if an age adjusted rate had been calculated.

Thus, compared with other studies the incidence of pleural mesothelioma in this study was low, but due to the small size this may be due to chance.

The distribution of mesotheliomas in the peritoneum and pleura has already been discussed by Browne and Smith. ${ }^{2}$ They compared the latency period, exposure time, association with asbestosis, severity of exposure, and fibre type and concluded that it was most probably the level of exposure, the opening of fibre bundles, and amount of contaminating dust-for example, from fillers-that would explain the high incidence of peritoneal to pleural ratio in some occupational groups. They found the evidence weak that fibre type would influence the ratio.

Another review indicated that the site of the mesothelioma mainly depends on fibre type. ${ }^{12}$ The review concluded that workers exposed to amosite had similar risks of peritoneal and pleural mesothelioma, almost all cases of mesothelioma attributed to chrysotile were pleural, whereas the evidence for crocidolite as a cause for peritoneal mesothelioma were inconsistent. However, different types of persistent fibres deposited in the peritoneum cause peritoneal mesothelioma in animal experiments. ${ }^{13}$ Furthermore, the importance of the dose gets some support from the finding that eronite, a very persistent fibre, mainly causes pleural mesothelioma at a high rate at very low exposure levels in humans. ${ }^{14}$

OTHER SITES OF CANCER

There also seems to have been an increased risk of cancer of the pancreas among these insulation workers. Some of these cases may be misclassified peritoneal mesotheliomas. A United States study of insulation workers showed that of 49 cases of pancreatic cancer among insulation workers 26 were reclassified after a review of the diagnosis; 16 of the reclassified cases were classified as peritoneal mesothelioma. ${ }^{15}$ Furthermore, the increased incidence of cancer at unknown sites of origin may also partly be misclassified tumours of the lung or peritoneum. Some other studies have indicated an increased risk of cancer of the stomach, large bowel, and rectum in workers exposed to asbestos. ${ }^{7}$ This study has little power to detect a moderate RR and there is no indication of an increased incidence of these sites.

ATTRIBUTABLE RISK

The increased mortality and cancer morbidity in these workers seem to be mainly caused by asbestos. The attributable risk during the 1980s and early 1990s was about $50 \%$ for death from all causes and cancer of all sites. Thus more than every second death and case of cancer could have been prevented if the exposure to asbestos had not occurred among these insulation workers.

This work was supported by the $\mathrm{J} \mathrm{K}$ Jubilee Fund in Gothenburg. 
1 Doll R, Peto J. Asbestos. Effects on health of exposure to asbestos. Health and Safety Commission. Her Majesty's Stationtos. Health and Safety Comm
ery Office, London, 1985.

2 Browne K, Smither WJ. Asbestos-related mesothelioma: factors discriminating between pleural and peritoneal sites. Br F Ind Med 1983;40:145-52.

3 Sandén Å, Järvholm B, Larsson S, et al. The risk of lung cancer and mesothelioma after cessation of asbestos exposure: a prospective study of shipyard workers. Eur Respir F 1992;5:281-5.

4 Sandén A, Näslund PE, Järvholm B. Mortality and morbidity of Swedish insulation workers. Scand 7 Work Environ Health 1984;10:207.

5 Berry G. The analysis of mortality by the subject-years method. Biometrics 1983;39:173-84.

6 Mattson B, Wallgren A. A completeness of the Swedish Cancer Register: non-notified cancer cases recorded on death certificates 1978. Acta Radiol 1984;23:1-9.

7 Selikoff IJ, Seidman H, Hammond EC. Mortality experience of insulation workers in the US and Canada 1943-76. ence of insulation workers in the US

8 Walker AM. Declining relative risks for lung cancer after cessation of asbestos exposure. F Occup Med 1984;26:422-4.
9 Mossman BT. Mechanisms of asbestos carcinogenesis and toxicity: the amphibole hypothesis revisited. Br f Ind Med 1993;50:673-6.

10 Folkhälsoinstitutet (National Institute of Public Health). Aktuellt om tobak. Tobaksstatistik 1980-95. (Tobacco statistics 1980-95). Stockholm: Folkhälsoinstitutet, 1996. (Tobaksprogrammets faktahäfte no 1 .)

11 Coggon D, Inskip H, Winter P, et al. Differences in occupational mortality from pleural cancer, peritoneal cancer, and asbestosis. Occup Environ Med 1995;52:775-7.

12 HEI-AR. Asbestosis in public and commercial buildings: a literature review and synthesis of current knowledge. Cambridge: Health Effects Institute-Asbestos Research, 1991.

13 Davis JM, Bolton RE, Miller GB, et al. Mesothelioma dose response following intraperitoneal injection of mineral fibers. Int f Exp Pathol 1991;72:263-74.

14 Baris YI, Simonato L, Artvinli M, et al. Epidemiological and environmental evidence of the health effects of exposure to erionite fibers: a four-year study in the Cappadocian region of Turkey. Int f Cancer 1987;39:10-17.

15 Selikoff IJ, Seidman H. Cancer of the pancreas among asbestos insulation workers. Cancer 1981;47:1469-73.

\section{Occupational and Environmental Medicine and the electronic age}

OEM has an Email address which is 100632.3615@compuserve.com. We welcome contact by Email, including letters to the editor. Many of our reviewers send us their reports by Email, helping to speed up the peer review process.
Our publishing system is now fully electronic, and authors are sending their revised copy to us on disk as well as paper. Watch for revised Instructions to Authors.

The Editor 